\title{
Wave Radiation of a Cycloidal Wave Energy Converter
}

\author{
S. G. Siegel \\ Atargis Energy Corporation, 1111 Lavender Way, Pueblo, Colorado, 81001, USA.
}

\begin{abstract}
Numerical results from a three-dimensional radiation model are presented where a Cycloidal Wave Energy Converter (WEC) is interacting with an incoming straight crested Airy wave. The radiation model was developed in response to experimental observations from 1:10 scale experiments which were conducted in the Texas A\&M Offshore Technology Research center wave basin. These experiments were the first investigations involving a WEC where three dimensional wave radiation effects were present due to the fact that the span of the WEC was much smaller than the width of the basin. The radiation model predicted the observed surface wave patterns in the experiment well, and showed that radiation induced wave focusing increased the recoverable wave power beyond the two-dimensional predictions for small WEC spans, while approaching the two-dimensional limit for very large spans. The numerical model was subsequently used to investigate the sensitivity of the WEC to misalignment between the incoming waves and the WEC shaft as well as the impact of a gap in the blade setup of a double WEC. For misalignment, the loss in efficiency was found to be strongly dependent on the ratio between WEC span and incoming wavelength, where short spans (on the order of one wave length or less) which are realistic for actual ocean deployment showed only minor reductions in efficiency, while very long spans were found to be more sensitive to misalignment. The blade gap in a double WEC setup was found to have a relatively minor effect (up to 30\%) on efficiency. Efficiency was found to either increase or decrease depending on the size of the gap.
\end{abstract}

Keywords: Wave Energy Conversion, Cycloidal Turbine, Deep Ocean Wave, Hydrofoil, Cycloidal Wave Energy Converter, Cost of Energy, Wave Radiation

\section{Introduction}

Among alternative energy sources, wave power is one of the most abundant sources on earth. The World Energy Council according to [1] has estimated the world wide annual amount of wave energy at 17.5 PWh (Peta Watt hours $=10^{12} \mathrm{kWh}$ ). This amount of energy is actually comparable to the annual world wide electric energy consumption, which is currently estimated at $16 \mathrm{PWh}$. Thus, wave energy has the potential to provide a large portion of the worlds electric energy needs, if it can be harnessed efficiently. In addition to the energy availability, wave energy has other advantages. Since a large portion of the worlds population lives close to the ocean shores, the distance between energy production and consumption is small, which reduces transmission losses and necessary investments in transmission lines. As opposed to other alternative energy sources like wind, stream and solar energy, the installation of wave power devices does not require use of already precious real estate. This makes wave energy an ideal energy source for efficiently providing renewable energy to densely populated coastal areas. Thus ocean waves have a tremendous potential to provide clean renewable energy. Further engineering aspects of wave power as an energy source are appealing as well. While the power density of both solar and wind in typical favorable sites is in the order of $1 \mathrm{kWm}^{-2}$ [2], wave power in a typical North Atlantic wave that was considered in a related paper [3] (wave height of $H=3.5 \mathrm{~m}$ and period of $T=9 \mathrm{~s}$ ) yields $108 \mathrm{kWm}^{-1}$ of wave crest. As shown there, a device extending about $40 \mathrm{~m}$ in the vertical direction can extract almost all of this wave energy, yielding a power density of about $2.7 \mathrm{kWm}^{-2}$ or more than two and a half times that of wind or solar power. If one considers the theoretical inviscid conversion limits for waves and wind, which are $100 \%$ for waves [4] and 59\% for wind [5], the accessible power density of waves is more than four times as large as that of wind. Furthermore, wave energy is available on a more consistent basis and can be better predicted in advance, therefore mitigating the need to back up a wave power plant with other conventional power sources, such as solar and wind energy. 


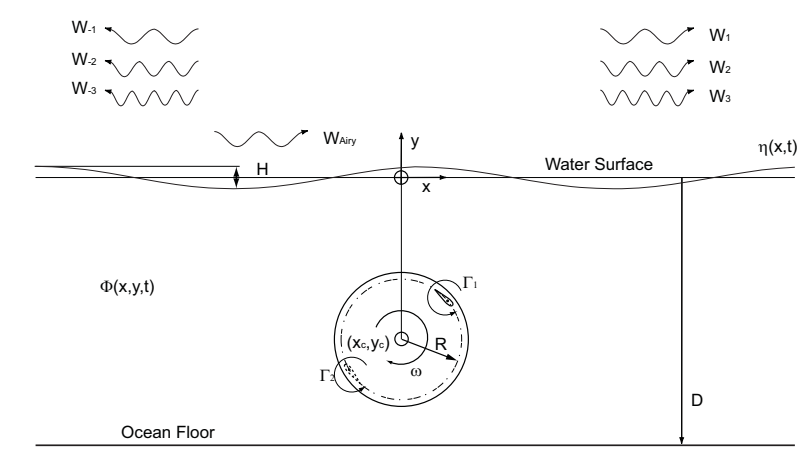

Figure 1: Cycloidal wave energy converter geometry and generated waves

\section{Motivation and Objectives}

Given the attractive features of wave energy as an alternative energy source, it has received significant attention in the scientific community over time. While a comprehensive review of all relevant publications would be prohibitively long, the reader is instead referred to comprehensive reviews published by McCormick [6], Mei [7] or, most recently, Cruz [8]. The following discussion will instead focus only on select sources most pertinent to the current work.

While wave energy as a resource may be free, the construction effort to harness it is a major expense and to a large degree determines the cost of energy being produced. As a less efficient WEC will need to be larger in size to extract the same amount of energy as a more efficient one, cost of energy is directly related to efficiency. Arguably, the most efficient WEC is one that can extract all of the energy from an incoming wave, and the class of wave energy converters that is able to achieve this is commonly referred to in literature as wave termination devices. There have been various wave termination designs reported in literature, with the most well known devices being the Salter Duck [9] and the Bristol or Evans Cylinder [10]. Both consist of a series of elements which are aligned parallel to the wave crests, in the case of the Salter Duck these are cam-shaped and floating on the surface, while the Bristol Cylinder is fully submerged. Both have been shown to be able to absorb an incoming wave completely. The wave energy is converted to electric power by means of a power-take-off system that is hydraulic in both cases. As both devices move at approximately the wave induced water velocity, the devices need to feature a large surface area to convert appreciable amounts of power. This increases construction cost, reduces storm survival odds and has ultimately motivated the investigation of the Cycloidal WEC described here. The fact that both devices require mooring to the ocean floor also hampers storm survival odds and precludes installation in very deep water.

A typical cycloidal wave energy converter as considered in this paper is shown in Fig. 1. It features one or more hydrofoils attached eccentrically to a main shaft at a radius $R$. While the shaft rotates, the pitch angle of the blades may be adjusted. This device operates at a rotational speed of the hydrofoil that is typically an order of magnitude larger than the wave induced water velocity, and employs the lift force of the hydrofoil to generate shaft torque directly. Using lift allows for a much smaller hydrofoil plan form area to be employed compared to the cross sectional areas of Duck and Cylinder, and generating shaft torque directly eliminates the need for a costly and inefficient hydraulic or linear generator type power take off system.

A single rotating hydrofoil was first investigated by Hermans et al.[11] both numerically and experimentally. While Marburg [12] reported very low wave energy conversion efficiencies (on the order of a few percent) in these experimental investigations, Siegel et al.[13] were able to show in simulations that with improved sizing of the WEC as well as by using synchronization of the rotation of the foil with the incoming wave, wave termination with better than $99 \%$ inviscid efficiency was possible. These numerical findings were confirmed by 1:300 scale experiments in 2011, as reported by Siegel et al.[14] where invisicid conversion efficiencies of greater than $95 \%$ were achieved. Both of these initial studies performed synchronization of the WEC with a numerically generated harmonic wave, or a paddle wave maker, respectively. Thus they did not require a feedback controller and estimator to succeed. A controller and estimator were for the first time successfully implemented by Jeans et al.[15] for irregular waves in a numerical simulation. Typical conversion efficiencies in this study were beyond $90 \%$ for a superposition of two harmonic waves, and around $80 \%$ for irregular waves following a Bretschneider distribution. At the same time, 


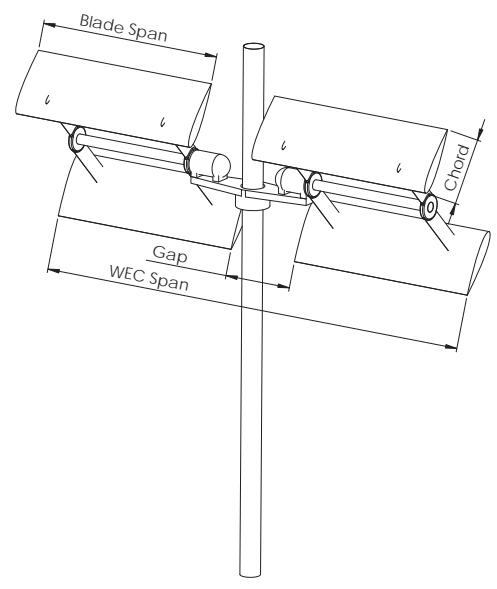

Figure 2: Sketch of Double Cycloidal Wave Energy Converter attached to a monopile

the controller and estimator were successfully tested in an experiment as reported in [16] where harmonic waves with different wave heights and frequencies were successfully cancelled, achieving efficiencies comparable to the earlier synchronization experiments that had a priori knowledge of the incoming wave. The performance of the feedback controller and estimator could thus be experimentally verified for the first time. Next, the WEC investigations were advanced by experimentally canceling both a superposition of two harmonic waves, as well as irregular waves following a Bretschneider distribution. This has been done in simulations reported by Jeans et al.[15], as well as experimentally validated by Siegel et al. [17] in a small 2D wave flume .

The first wave cancellation experiments in a wave tank where the span of the WEC was far smaller than the width of the tank were conducted in 2012 at the Texas A\&M Offshore Technology Research Center and established successful electricity production for the first time, see Fagley et al.[18]. Experimental observations also indicated the presence of 3D radiation effects, and an initial version of a numerical model described in Fagley et al.[18] found good agreement between experiment and numerical model. In this work, the radiation model is further improved and used to investigate the sensitivity of the WEC to angular offsets between WEC shaft and wave crest direction. It is of importance to predict the possible efficiency reductions as a result of this type of misalignment. It is also necessary to quantify the necessary accuracy of wave measurement equipment employed for alignment of WEC and incoming wave.

A second focus of this work is the investigation of the impact of a blade gap on the performance of the WEC. For a WEC attached to a monopile, as shown in Fig. 2, a gap between the left and right WEC blades exists in order to accommodate the central mount. It is thus of interest to investigate the impact that this gap has on the overall WEC radiation pattern.

\section{3D Wave Model}

The surface elevation $\eta_{c}$ of a circular wave can be described as, $\eta_{c}(x, z, t)=H(r) \sin (\omega t-k r)$ with $\mathrm{x}$ and $\mathrm{z}$ the wave travel and wave normal coordinates, $\mathrm{k}$ the wave number, $r=\sqrt{\left(x-x_{0}\right)^{2}+\left(z-z_{0}\right)^{2}}$ the distance from the wave center, $\mathrm{T}$ the wave period and $\mathrm{t}$ time. If this wave is to conserve energy as it radiates outward, the wave height $\mathrm{H}$ has to decrease with distance from the wave generator. If the initial wave height is specified as $H_{0}$ at a finite distance $r_{0}$ from the center, 


$$
H(r)=\sqrt{\frac{H_{0}^{2} r_{0}}{r}},
$$

the wave does conserve wave energy resulting in a reduced wave height as the wave propagates away from the wave center at a $1 / r$ relationship.

The initial version of the numerical radiation model employed in this study has been reported in Fagley et al.[18]. There, the WEC generated wave was synthesized from a superposition of semi-circular waves according to Eq. 1. A typical semicircular wave of this type features equal wave height for all azimuthal locations at a given distance from the source. Since the WEC produced single sided waves as reported in Siegel et al. [13], the left two quadrants were set to zero. This led to an abrupt transition from zero to full wave height along the $x=0$ axis, and caused discrepancies between numerical model and experimental observations and wave gauge measurements. To improve this behavior, the present simulations use a modified circular point source,

$$
\begin{aligned}
H(r, \theta) & =\sqrt{\frac{H_{0}^{2} r_{0}}{r}} \cos \theta \\
\theta & =\arctan \left(\frac{z-z_{0}}{x-x_{0}}\right),
\end{aligned}
$$

where the wave height was modulated in the azimuthal direction as a function of the angle $\theta$ between wave travel direction (x-axis) and spatial location at the surface. The resulting surface wave pattern is shown in Fig. 3 and featured a maximum wave height in the positive wave travel direction, and zero wave heights in the positive and negative wave crest directions. The physical argument why this distribution was appropriate for modeling the WEC wave is related to the travel speed of the hydrofoil in the wave propagation direction: Along the $\mathrm{x}$ axis, the hydrofoil moved with the rotational speed in the direction of the wave propagation (the $\mathrm{x}$ axis). Conversely, along the $\mathrm{z}$ Axis the hydrofoil travel velocity and the wave propagation direction (the $\mathrm{z}$ axis) were perpendicular, thus creating no wave in that direction. For all angles in between, a cosine function described the component of the hydrofoil velocity aligned with the wave propagation direction. Beyond this physical argument, it was shown [19] that this model provided better agreement with experimental data. It also avoided the abrupt wave height transitions of the semi-circular model.

It was possible to use several semi-circular waves to approximate the wave pattern caused by more complex wave generators. For a WEC, the waves generated could be approximated as a sum of individual semi-circular wave generators arranged along a line of finite length equal to the span S of the WEC blades, as expressed by

$$
\eta_{W E C}(x, z, t)=\sum_{n}^{N} \eta_{c n}, x>0 .
$$

Single sided wave generation was again assumed and assured by setting the WEC wave to zero for negative $\mathrm{x}$ coordinates, and was experimentally verified in as described in the following chapter. For a WEC perfectly aligned with the incoming wave, the $\mathrm{N}$ circular wave generators were located between $z=-S / 2$ and $z=S / 2$ along $x=0$. A number of $N=25$ individual circular waves was found to produce converged results. The strength of each circular wave generator used to discretize the WEC wave generation along the span of the hydrofoil was approximated by either a constant or an elliptical distribution according to the following equation:

$$
H_{0}(z)=H_{c} \sqrt{\left(1-\left(\frac{z}{2 S}\right)^{2}\right.} .
$$

This modeled the lift distribution along the span of the foil which caused a wave height that was proportional to the local circulation which was shown in two dimensional potential flow simulations in Siegel et al. [13]. To assure that the maximum wave height at the center of the foil was $H_{c}$, the overall generated wave height was renormalized to the value specified. The wave field generated by the WEC was then superimposed with the wave field generated by the incoming wave. The incoming wave in this investigation was a long crested Airy wave traveling in the positive 


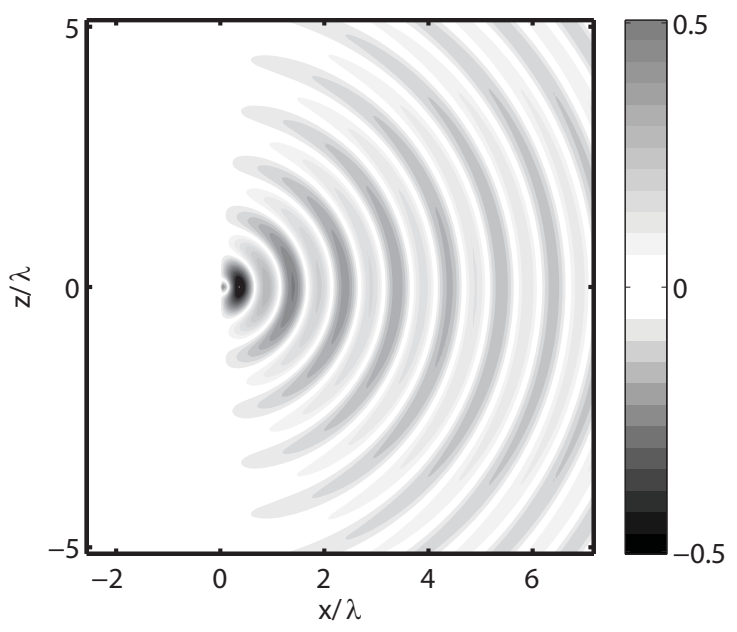

Figure 3: Surface Elevation of a fundamental circular wave with cosine modulated radial wave height

$\mathrm{x}$ direction modeled as, $\eta_{A}(x, z, t)=H \sin (\omega t-k x)$ The final surface elevation was then calculated by superimposing the incoming airy wave with the wave generated by the WEC.

It was not a priori obvious which WEC center wave height $H_{c}$ would cause optimal WEC efficiency. In general, if $H_{c}$ was too small, incomplete wave cancellation would lead to less than optimal efficiency. On the other hand, if $H_{c}$ was chosen too large, the wave radiated by the WEC more than canceled the incoming wave, leading to reduced efficiency due to the power of the remaining wave being radiated away. In between these extreme cases an optimal value for $H_{c}$ exists, as will be shown in the results section. Throughout this paper, a parameter study was conducted for each case investigated, and only the optimal value of $H_{c}$ was reported for all data presented. It should also be noted that a given WEC design may not be able to achieve a desired $H_{c}$ height and thus may not be able to achieve optimum efficiency. The wave height that can be canceled by a given WEC is dependent on the WEC size $2 R / \lambda$ as well as the hydrofoil circulation $\Gamma$ (see Siegel et al.[13]). The circulation that can be produced by a given hydrofoil in turn depends on its chord length, rotational speed as well as maximum lift coefficient. These interdependencies lead to a complex relationship between the wave parameters wavelength and wave height, as well as the WEC design parameters radius, foil shape and foil chord and are the subject of a separate study reported in Siegel [20]. Therefore, the maximum theoretically obtainable efficiencies are reported here independent of the ability of a given WEC design to achieve the necessary center heights or not for a given incoming wave. The efficiencies reported here can thus be considered wave radiation based upper bounds which cannot be exceeded, but may be reached depending on WEC design.

\subsection{Control Volume analysis}

The wave field resulting from the interaction of incoming Airy wave and the waves generated by the semi-circular waves used to model the WEC was used to determine the overall energy absorbed by the WEC. Using a control volume that enclosed the WEC completely, the fundamental waves entering and leaving that control volume could be calculated. To ease calculations, the control volume chosen was a rectangle aligned with the coordinate system axes. The circular waves were decomposed in a $\mathrm{x}$ and $\mathrm{z}$ component. Thus, the portion of each circular wave leaving the control volume boundaries could be calculated at each location along the boundaries. For an Airy wave, wave power $P$ per unit length can be calculated as

$$
P=\frac{\rho g}{8} H^{2} C_{g},
$$

with $C_{g}$ being the wave group velocity, $\rho$ the density of water and $g$ the gravity constant. Thus the wave power traversing a control volume boundary extending in the $\mathrm{z}$ direction from $z_{1}$ to $z_{2}$ could be calculated by 


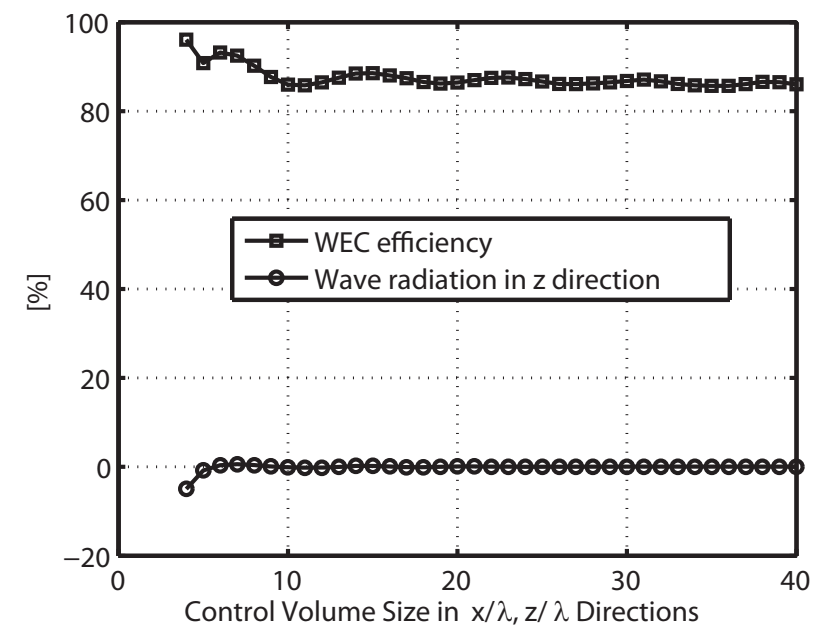

Figure 4: Convergence of control volume analysis results as a function of control volume size

$$
\begin{gathered}
P=\int_{z_{1}}^{z_{2}} \sum_{m=1}^{M} P_{m x}(z) d z \\
P_{x}(z)=\frac{\rho g}{8}\left(H_{x}^{2}+H_{z}^{2}\right) C_{g x} .
\end{gathered}
$$

The subscripts indicate the vector component of the respective quantity in that direction. These equations can be modified to calculate the power traversing across a horizontal control volume boundary by swapping the subscripts $\mathrm{x}$ and $\mathrm{z}$. The overall amount of power extracted by the WEC could then be calculated by choosing a closed rectangular control volume and accounting for all wave power being transported across its boundaries. To determine the efficiency with which the WEC extracted energy from the waves, a reference quantity equal to the wave power of the Airy wave times the overall span $S$ of the WEC was used. Thus, if the control volume analysis showed that this amount of energy was extracted from the waves, the efficiency was unity or $100 \%$. Any efficiency larger than this indicated that radiation induced wave focusing was encountered, while efficiencies below 100\% indicated losses due to waves being radiated in the wave crest direction, or increases in wave height due to constructive interaction between WEC wave and incoming wave as a result of ill matched phase. The control volume analysis was subjected to a convergence study in both control volume size, as well as discretization interval. Fig. 4 shows the WEC efficiency and wave power radiated in the $\mathrm{z}$ direction as the size of the control volume is increased in both directions simultaneously. Based on the data shown a control volume of equal extent in the wave travel (x) and wave crest (z) directions of 20 wave lengths in size was chosen. A similar investigation for discretization interval (not shown) indicated that a discretization of $1 / 10$ of a wave length was required for convergence. Small residual periodic fluctuations in the resulting wave power were averaged over one period in space. The overall accuracy of the control volume analysis is estimated to be better than $\pm 2.5 \%$ in conversion efficiency based on the convergence data.

\section{Experimental Setup}

\subsection{Wave Basin}

All experiments reported here were conducted at the Texas A\&M University Offshore Technology Research Center (OTRC) wave basin. The basin was $45.7 \mathrm{~m}$ in length, 30.5m in width and had a water depth of 5.8m. A set of 48 hinged flap wave makers, individually controlled by hydraulic cylinders, spanned one of the short sides of the basin. At the other end, a progressive wave absorber consisting of stacked screens acted as an artificial beach to reduce reflection of incoming waves. The wave maker had a maximum design wave height of $H_{W M}=0.9 \mathrm{~m}$ at a wave period of $T=2.5 \mathrm{~s}$. 


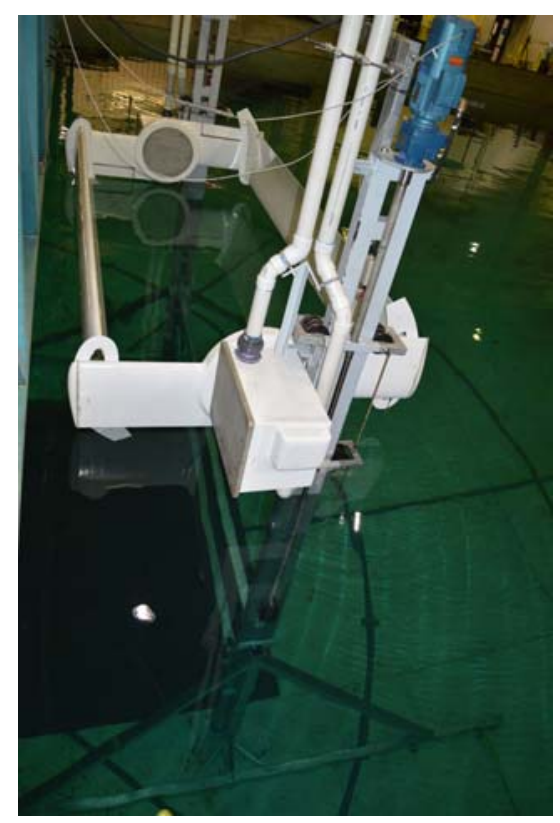

Figure 5: WEC 1:10 scale model installed in OTRC wave tunnel. The WEC is lifted above the water line during installation

This wave was a deep water wave with a wave length of $\lambda=9.75 \mathrm{~m}$ and thus determined the model scale of $1: 10$ compared to a full scale deep ocean design wave for the WEC. While current and wind generators were available, they were not used in the present investigation. In order to investigate the surface wave pattern caused by the WEC and its interaction with incoming waves, a set of 10 wire type capacitive wave gauges were installed at a distance of $8 \mathrm{~m}$ from the shaft of the WEC. The locations of the wave gauges are shown in Fig. 7. The wave gauge calibration was estimated to be accurate to within $1 \mathrm{~mm}$ of water level. For all waves generated, a calibration was performed with the model removed from the water in order to assure that the wave heights at the model location matched the target values. For this calibration, a wave gauge was placed at the center of the model.

\subsection{Cycloidal WEC Model}

The WEC model, shown in Fig. 5, was designed specifically to efficiently interact with the wave climate in the OTRC tunnel. It featured two hydrofoils with a chord length $c=0.75 \mathrm{~m}$ and $\operatorname{span} S=4.5 \mathrm{~m}$. The hydrofoils had a NACA0015 cross section with a curved camber line that matched the radius $\mathrm{R}=1 \mathrm{~m}$ at which they were attached to the main shaft. End discs with a height of $10 \mathrm{~cm}$ were installed at the ends to minimize tip vortex losses. The hydrofoils were attached to a split main shaft by means of two struts located at the ends of the hydrofoils. The struts were equipped with linear actuators allowing for control of the hydrofoil pitch. Each of the struts was streamlined with a NACA 0015 hydrofoil section. The main shafts were connected to two individual motor/generator units installed in water proof enclosures. These consisted of 3 Phase 230V AC 3.7kW asynchronous motors coupled to 80:1 gear boxes. The shafts were instrumented to allow for direct measurement of the shaft torque by means of two load cells. The load cells were calibrated by the manufacturer and delivered an accuracy of $0.05 \%$ of their full scale reading, which coincided with the maximum motor torque. The shaft angle was measured directly using incremental rotary encoders for position feedback. The motors were operated by four quadrant capable inverters allowing for software control of torque, speed and position. These inverters were also electronically linked to allow for synchronous rotation of both shafts. Breaking resistors allowed for dissipation of the regenerative power produced during wave power extraction. As the inverters were operated over a CANOpen network, pertinent parameters such as shaft position, velocity and motor current were reported back to the control computer and logged in sync with all other data. The motor current readings had a resolution of $0.1 \%$ of the nominal motor current. While the motors themselves were installed in water proof enclosures, the respective inverters were located in an electronics rack located on the bridge spanning the 
tunnel. This rack also accommodated the control computer used for feedback control of the WEC as well as for data acquisition and logging.

A mounting frame allowed for attachment of the WEC to hard points in the tunnel floor. While this provided a fixed horizontal positioning of the WEC in the tunnel, the WEC model itself could be traversed in the vertical direction by means of two lead screws operated by gear motors, which allowed access to the model when lifted above the water line and adjustment of submergence depth of the model during operation. The range of motion was $-1.8 m<y_{c}<1.2 m$ and could be controlled with an accuracy of a fraction of a millimeter. Fig. 5 shows the WEC model installed on the frame in the OTRC tunnel, while lifted out of the water.

All communication with motor controllers, pitch actuators and data acquisition was accomplished through a CAN bus network under a CANOpen software protocol. The feedback control software was run on a PC and written in LabVIEW, operating at an update rate of $20 \mathrm{~ms}$. All data logged during the experimental runs was synchronized using the CANOpen SYNC message ensuring that the measurements were taken at the same instants of time. The SYNC message also provided hardware based timing for the control loop providing feedback to the WEC.

\section{Results}

The following chapters outline results and observations from the experiment, followed by a comparison between the experimental data and the numerical result. The final two subsections of this chapter present numerical results for interaction of an incoming wave oriented at an angle to the WEC shaft, and a double WEC with a center gap between left and right blades.

\subsection{Experimental Observations}

Beyond shaft power and reactive force measurements reported elsewhere [18], the OTRC wave tunnel was equipped with 10 capacitive wave gauges for this experiment. These were arranged equidistant at a distance of $8 \mathrm{~m}$ to the WEC, which was placed in the center of the wave basin as shown in Fig. 7. The WEC could be operated both in wave cancellation mode where energy was extracted from an incoming wave, or in wave generating mode where motor power was used to rotate the blades which subsequently created a single sided wave as reported in Fagley et al. [18]. The color contour overlay in Fig. 7 shows an incoming wave being canceled by the WEC, with the wave traveling in the positive $\mathrm{x}$ direction. A typical radiation pattern due to the interaction between the WEC generated wave and the incoming wave can be seen to the right of the WEC, which is centered at $\mathrm{x}=0$ and $\mathrm{z}=0$.

This WEC radiation pattern could be observed in the wave tank experiment, Fig. 8 shows both the incoming and WEC waves impacting the screen system designed to prevent wave reflections. As the upper end of the screens provides a visual reference to visually gauge the water elevation at the screens, the modulated wave pattern across the tunnel can be well observed. This pattern indicates interaction of the WEC with the incoming waves, in the process of which wave energy is extracted. Without the WEC present or with the WEC at rest (not shown), the waves impacting the screens form a straight line of constant surface elevation across the wave basin, which fluctuates in height with the passage of each wave.

Thus the surface elevation observation shown in Fig. 8 provided qualitative evidence of wave energy extraction, while the next chapter compares quantitative data from both experiment and numerical simulation.

\subsection{Validation of Radiation Model}

In order to establish the validity of the numerical model beyond the first order rationale underlying its derivation as outlined in the numerical setup chapter, wave gauge data from the experiment was compared to predictions from the numerical model. Fig. 9 shows data from a wave generation experiment where no incoming wave was present, in comparison to the numerical model predictions. It can be seen that all wave gauge measurements located down-wave from the WEC agree well with the numerical model. In the experiment it was not possible to determine the $H_{c}$ value, since it was impacted by a local wave pattern around the WEC which is not expected to agree with the numerical model. This local wave pattern extends for about $1 / 2$ wave length and has subsided at the wave gauge locations. Instead, a single measurement run for each wave length was used to establish a $H_{c}$ scaling factor based on the center wave gauge (\#5). This scaling factor was then used to scale all other data at all wave gauge locations, a run not used for scaling factor determination is shown in Figure 9 (as can be seen by the small height difference at the center 


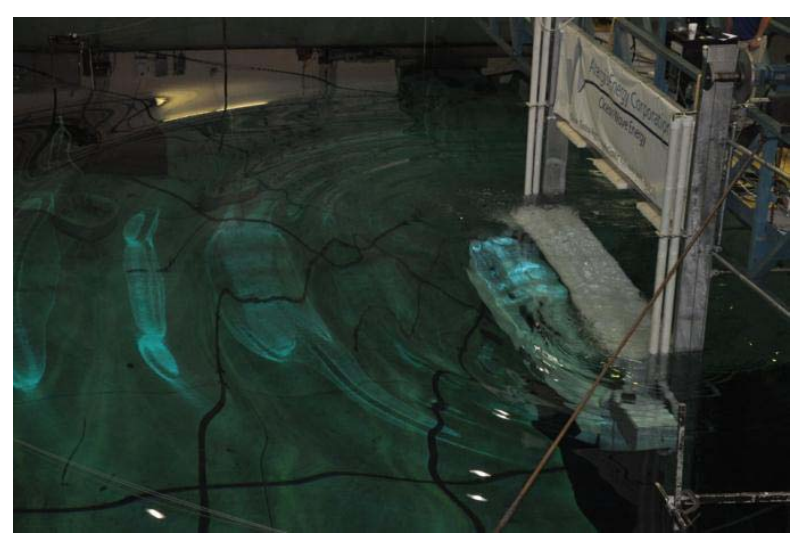

Figure 6: Picture of water surface pattern for a wave generation experiment. The WEC model is located underneath the banner, and the counter clockwise rotation causes waves traveling right to left to be generated

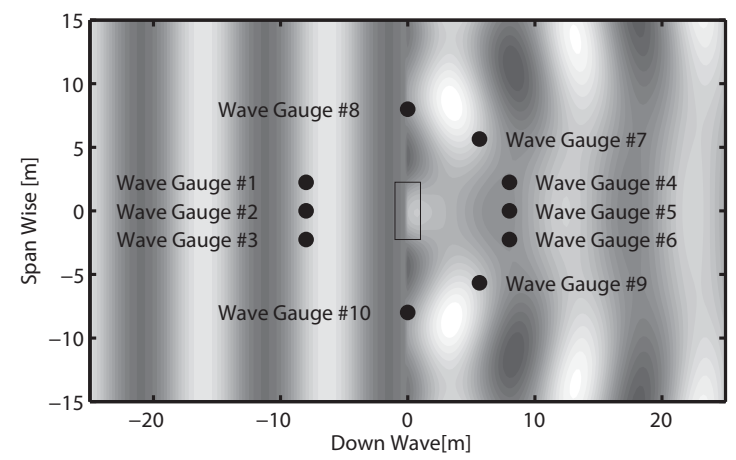

Figure 7: Instantaneous surface elevation for a wave cancellation simulation. WEC model is shown to scale as a rectangle, and the wave gauge locations are shown.

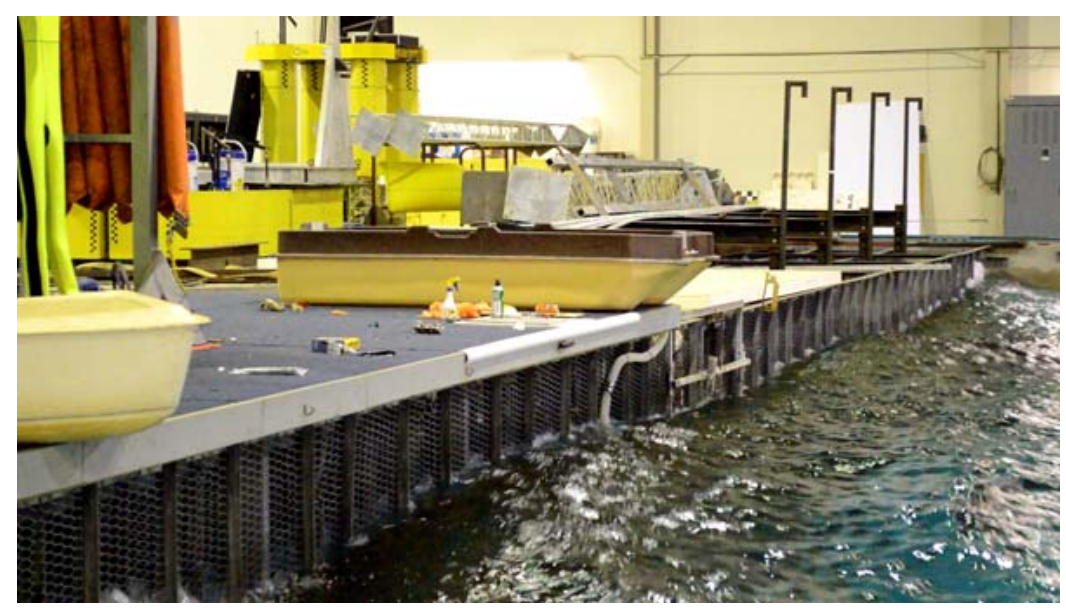

Figure 8: Picture of waves impacting screens at the end of the test section during an experimental wave cancellation run 


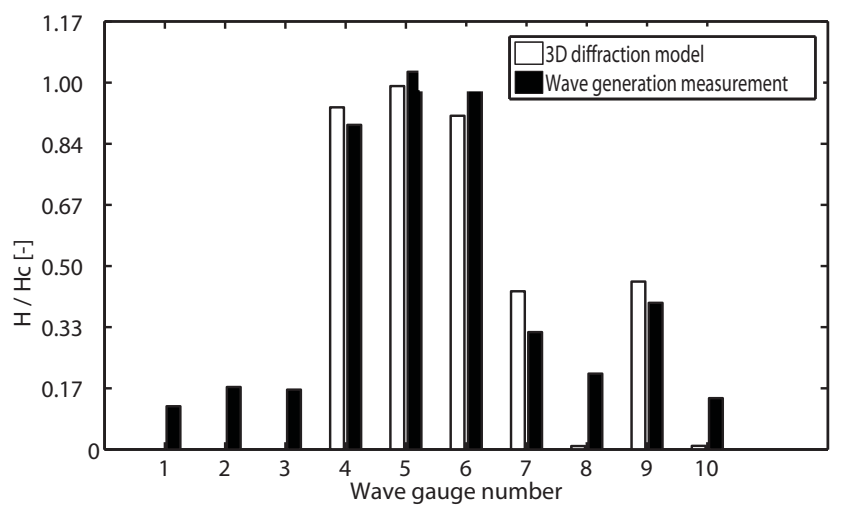

Figure 9: Comparison of wave heights from experimental measurements to predictions from the 3D Radiation model

wave gauge location). The numerical model data shown in Fig. 9 employed a cosine modulation of the point sources, which improved the match between numerical model and experimental data significantly compared to earlier results presented in Fagley et al.[18] where a constant azimuthal wave height was assumed. The improvement was most pronounced for wave gauges \#7 and \#9, which were located down-wave at an angle of 45 degrees from the tip of the WEC blade. In all portions of the wave field that were dominated by the WEC-wave interaction (i.e. wave gauges \#4 - \#9), the agreement between numerical model and measurements can be seen to be very good.

The largest discrepancies between measurements and numerical model can be observed up-wave of the WEC, where wave reflections in the experiment led to larger wave heights than what was predicted by the numerical model which assumed these to be zero by design. The experimental measurements down-wave of the WEC showing the largest discrepancies were at wave gauge locations \#8 and \#10, where the measurements were again larger than the numerical estimates. These were similar in magnitude to those observed up-wave of the WEC, and thus most likely caused by reflections just as those up-wave of the WEC.

While one particular data set is shown, the agreement between experiment and numerical simulation was improved for all experimental data by employing cosine azimuthal modulated point sources. The data set shown in Fig. 9 is representative of all measurements taken. Further comparison between experiment and numerical model was made and reported in Fagley et al.[18], which is why the present comparison for brevity is limited to demonstration of the improvement due to the cosine modulation.

\subsection{Radiation Efficiency for aligned WEC and Waves}

The initial use of the radiation model was to develop an understanding of the impact of the WEC span on the observed radiation pattern. Figures 10,11 and 12 show instantaneous snapshots of the water surface elevation for three different non-dimensional WEC spans of $S / \lambda=0.5, S / \lambda=1.0$ and $S / \lambda=3.0$, respectively. The radiation pattern shown in Fig. 10 corresponded closely to the design case of the experiment. While all three wave patterns featured a wake down-wave of the WEC, the wakes were distinctly different in their shape. For short spans, the wake spread at a large angle, while at the larger spans the wake became almost constant in width as it traveled down-wave.

With an azimuthal modulation of wave height introduced to the numerical model, the initial investigation focused on development of an understanding of the impact of this modulation on wave energy conversion efficiency. Fig. 13 compares the efficiency of two different radiation models (constant or top hat, and cosine modulated) along with two different span wise lift distribution models (again constant or top hat, and elliptical as described by Eq. 4). While the ratio between the incoming wave height and the wave height generated by the WEC at its center was varied, all other parameters were kept constant.

It could be observed that the top hat lift distribution led to a higher maximum efficiency regardless of radiation pattern and at a relatively small height ratio. This could be attributed to the good match between the incoming (constant height along the crest) wave and the equally constant wave generated by the WEC. The two simulations using an elliptical lift distribution required a higher height ratio to achieve optimal wave cancellation efficiency, and 


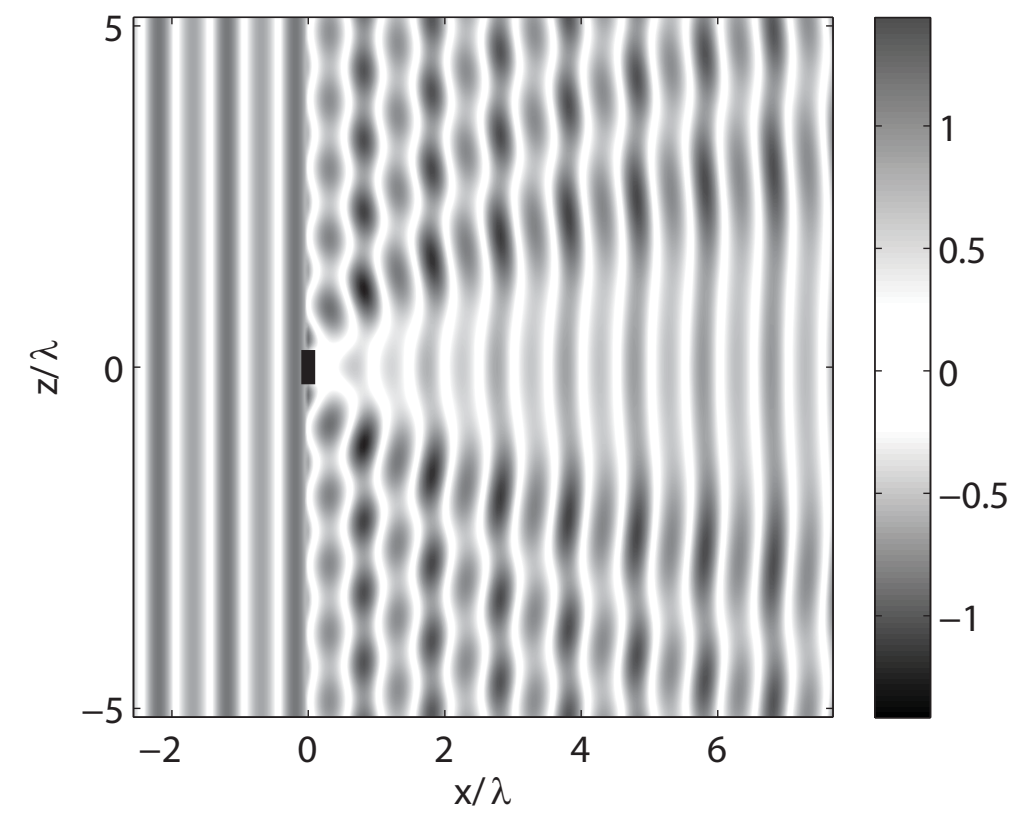

Figure 10: Surface Elevation during wave cancellation with a WEC of size $S / \lambda=0.5$ at angle $\alpha=0^{\circ}$ to the waves

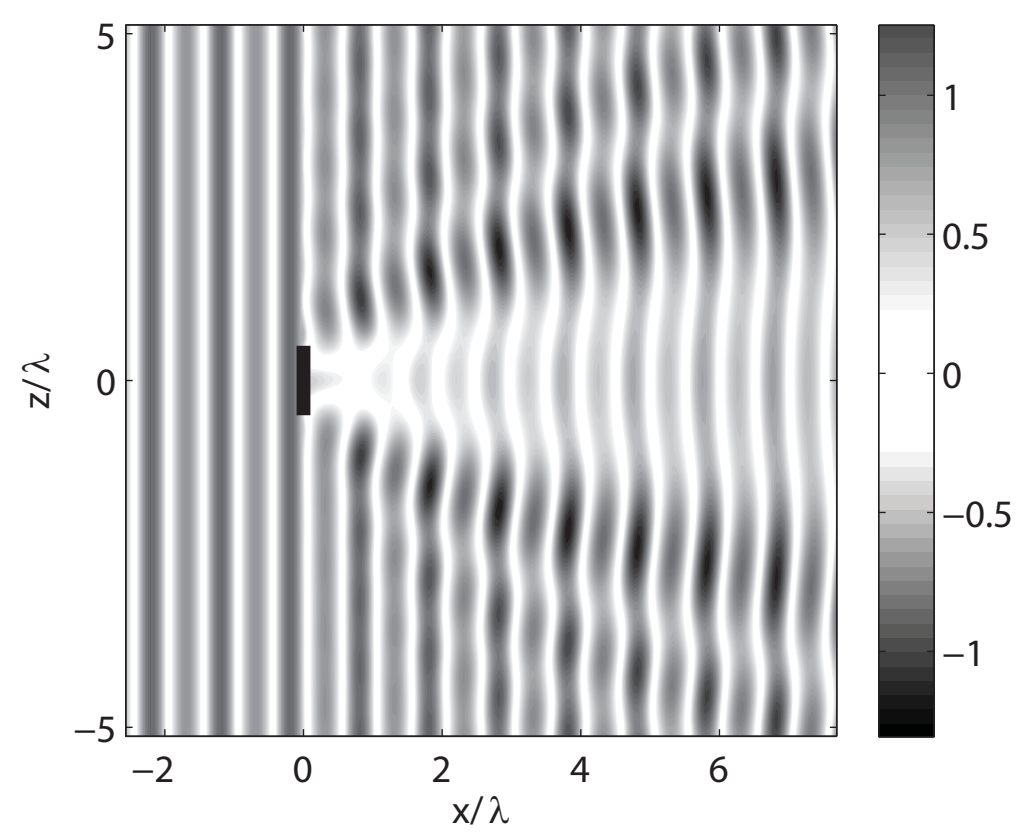

Figure 11: Surface Elevation during wave cancellation with a WEC of size $S / \lambda=1.0$ at angle $\alpha=0^{\circ}$ to the waves 


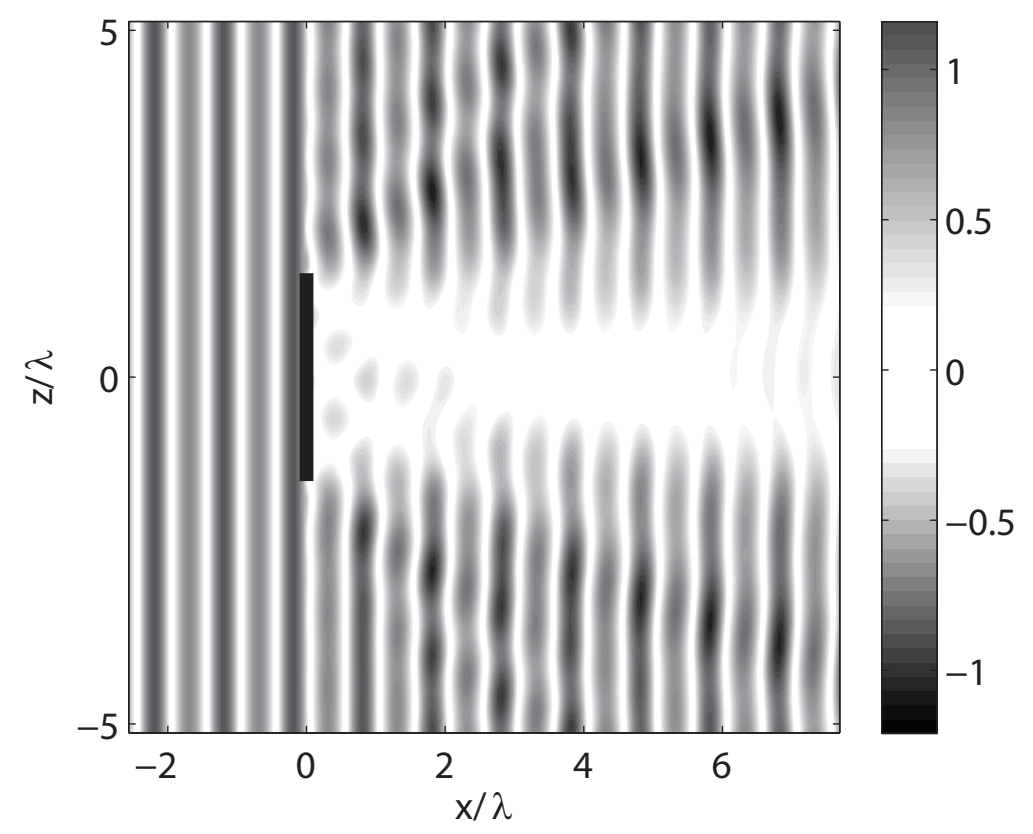

Figure 12: Surface Elevation during wave cancellation with a WEC of size $S / \lambda=3.0$ at angle $\alpha=0^{\circ}$ to the waves

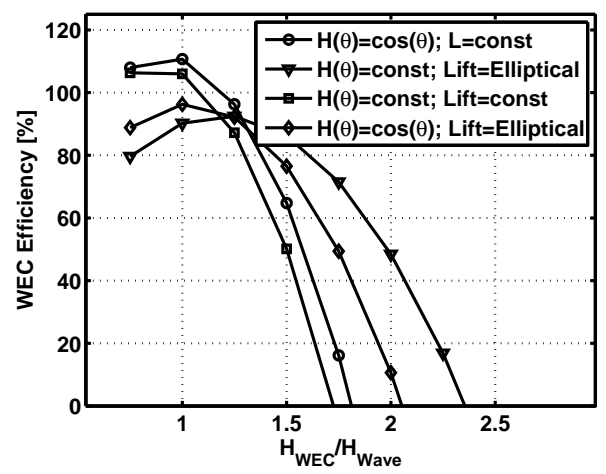

Figure 13: Wave Cancellation efficiency as a function of the ratio between incoming wave height and center height of the WEC generated wave. $S / \lambda=2, T=2.5 \mathrm{~s}$ 


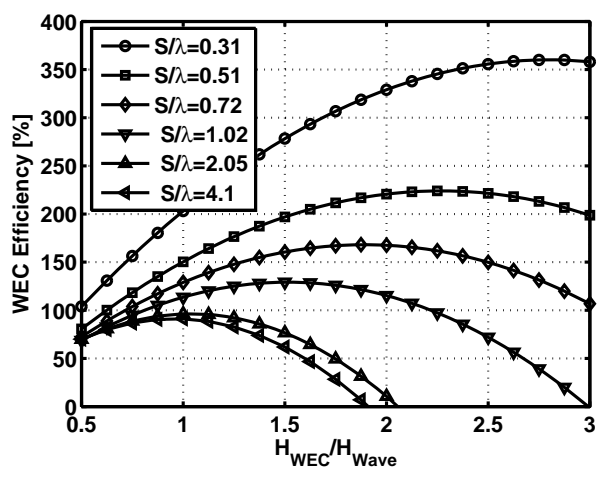

Figure 14: Wave cancellation efficiency and WEC Wave Height as a function of the ratio between incoming wave height and center height of the WEC generated wave for different Span to Wavelength Ratios. $T=2.5 \mathrm{~s}$

did not achieve the same efficiency but rather fell short by about 10 percent compared to the constant lift distribution. The higher height ratio correlated well with the smaller average wave height produced by an elliptical lift distribution, which was reduced by a factor of $\pi / 4$. From the data in Fig. 13 it could be concluded that a more realistic lift distribution (where an elliptical lift distribution constitutes an optimal situation in terms of hydrofoil induced drag) carried a penalty of about $10 \%$ in efficiency, compared to a top hat distribution that was only achievable in a theoretical study like the present one but could be seen to be favorable in terms of radiation efficiency. The impact of the radiation distribution on efficiency was less prominent, and at peak efficiency only caused a difference of a few percent. The cosine radiation distribution however could be seen to be favorable by a small margin for both lift distributions.

The data presented in all following figures was obtained using the cosine radiation distribution along with the elliptical lift distribution. In order to determine the optimal wave height ratio for a range of different span to wavelength ratios, a parameter study was conducted. Fig. 14 shows that there existed an optimal height ratio for each WEC span. This height ratio was larger for smaller spans, and also resulted in an overall larger efficiency. For small WEC spans, efficiencies in excess of $350 \%$ could be observed, indicating strong radiation and wave focusing effects. These results were in excess of our earlier findings using a constant radiation distribution. This was due to the fact that less wave power was lost in the span wise direction in the present cosine modulated numerical model, compared to the original constant radiation model. For very large WEC spans, however, the peak radiation efficiency dropped below $100 \%$. This was most likely caused by the non-uniform span wise lift distribution.

For control design purposes, the relationship between WEC Wave height and WEC span for optimum power extraction was of interest. Fig. 15 shows this relationship along with the resulting optimal efficiency. Both required WEC wave height and resulting efficiency were almost identical and reflected the underlying conservation of power of the numerical model.

In summary, the results of the cosine modulated radiation distribution surpassed those presented earlier for a constant azimuthal radiation model. A constant hydrofoil lift distribution however would (if it were achievable) perform better than a more realistic elliptical lift distribution.

\subsection{Radiation Efficiency for WEC and Waves at an angle}

The interaction between a WEC and an incoming straight crested wave always led to a symmetric radiation pattern, if the WEC shaft was aligned with the wave crests. A typical surface wave pattern is shown in Fig. 11 for a span equal to one wavelength. The angle of the first maximum of the wave crests relative to the wave travel direction was a direct function of the span to wavelength ratio, as was shown in Fagley et al. [18]. While perfect alignment is obviously the optimal configuration for this type of wave energy converter, this chapter explores the impact that misalignment has on WEC performance, as well as on the wave radiation pattern.

With all other parameters kept the same, the surface radiation wave patterns shown in Fig. 16 and Fig. 17 show the effects of an angle of $20^{\circ}$ and $45^{\circ}$ between WEC shaft and incoming wave crests, respectively. The WEC was rotated about its center for these investigations, which led to a positive phase error at one tip of the WEC, and an equal 


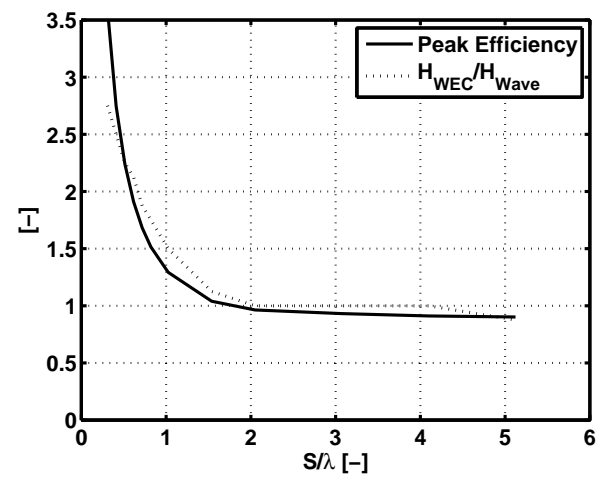

Figure 15: Optimum wave cancellation efficiency and WEC Wave Height as a function of the ratio between WEC Span and wavelength. $T=2.5 \mathrm{~s}$

magnitude but opposite sign phase error at the other tip due to the displacement of the tip relative to the $\mathrm{z}$ axis. The resulting radiation pattern show undisturbed waves in the upper portion of the wave field, and short crested waves in the lower portion. In both cases the wake in the wave pattern was not as pronounced as in the aligned situation shown in Fig. 11.

A parameter study where both WEC span as well as the angle between the incoming wave and the WEC shaft were varied was conducted next. Fig. 18 shows that there is a dramatic impact of the WEC span on the resulting efficiency loss for a given angle, with larger spans causing a larger reduction in efficiency.

A study was conducted varying the wave height ratio between incoming wave and wave generated by the WEC for all angles shown in Fig. 18. While not shown, varying the height ratio did not improve the efficiency. Similarly, the feedback phase between the incoming wave and the WEC wave was varied in a second study to investigate if a different phase than what was optimal for zero angle would be advantageous. This study also found no improvement in efficiency for variations in feedback phase. As the phase at the center of the WEC was identical for all angles to that of the zero angle situation, and the left end of the WEC had a phase lag equal in magnitude and opposite in sign to the right end of the WEC, this behavior was to be expected since the average phase mismatch would only increase for different feedback phases.

\subsection{Double WEC Radiation Efficiency}

Two typical wave radiation patterns of a double WEC with a center gap between the blades are shown in Fig. 19 and Fig. 20. While the radiation pattern of the double WEC with smaller gap size shown in Fig. 19 is very similar to a radiation pattern of a WEC without a central gap, the larger gap size shown in Fig. 20 shows a large residual wave down-wave of the gap, indicating that the larger gap allows for wave energy to be lost in this setup compared to a single WEC of same span.

Results from a parameter study where the gap size was varied while keeping the overall span constant is shown in Fig. 21. For the chosen span, there is a modest increase in efficiency as the gap size is increased. However, a much larger increase in required wave height can be seen, if compared to a single WEC. This effect can be explained by the shorter and shorter blade span that needs to be compensated for by a larger and larger wave height as the gap size is increased.

Results from a comprehensive parameter study showing the impact of both gap size as well as span on efficiency are summarized in Fig. 22. All results were normalized by the efficiency achieved for a given $S / \lambda$ ratio with no gap. The radiation efficiency for all $S / \lambda$ values of less than two can be seen to cause a monotonous increase in radiation efficiency with gap size. For a ratio of $S / \lambda=2$ a distinct optimum gap size exists around $G / \lambda=0.55$, beyond which the radiation efficiency decreased again as the gap size was increased further. While the radiation efficiency for all gap sizes was above the efficiency for a WEC without gap up to and including $S / \lambda=2$, this finding did not hold for larger values of $S / \lambda=3$ and beyond. In fact, for very large span to wavelength ratios of $S / \lambda=4$ and beyond any gap led to a decrease in radiation efficiency. The physical reason for these changes in efficiency are related to the passage 


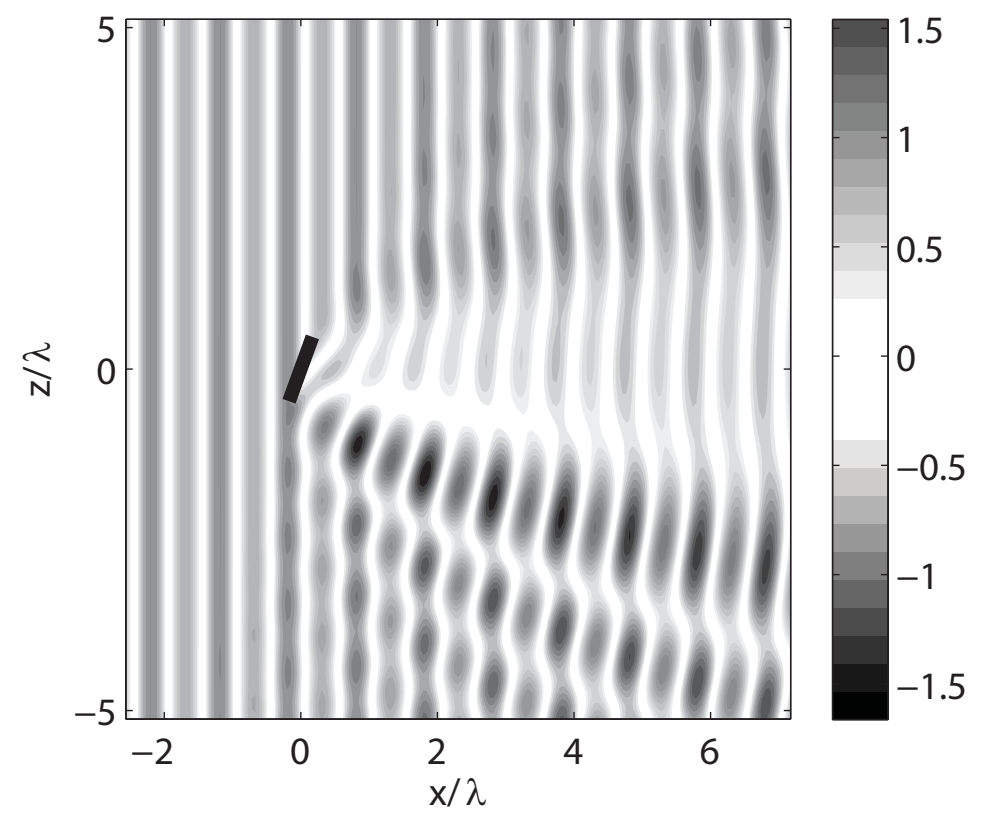

Figure 16: Surface Elevation during wave cancellation with a WEC of size $S / \lambda=1$ at angle $\alpha=20^{\circ}$ to the waves

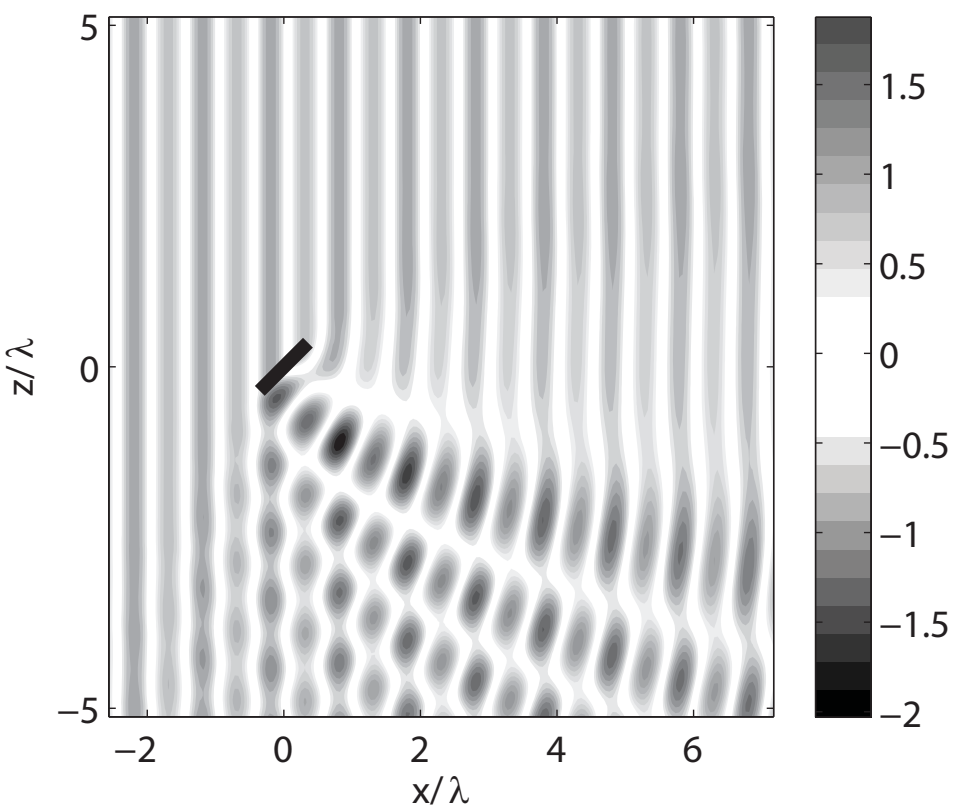

Figure 17: Surface Elevation during wave cancellation with a WEC of size $S / \lambda=1$ at angle $\alpha=45^{\circ}$ to the waves 


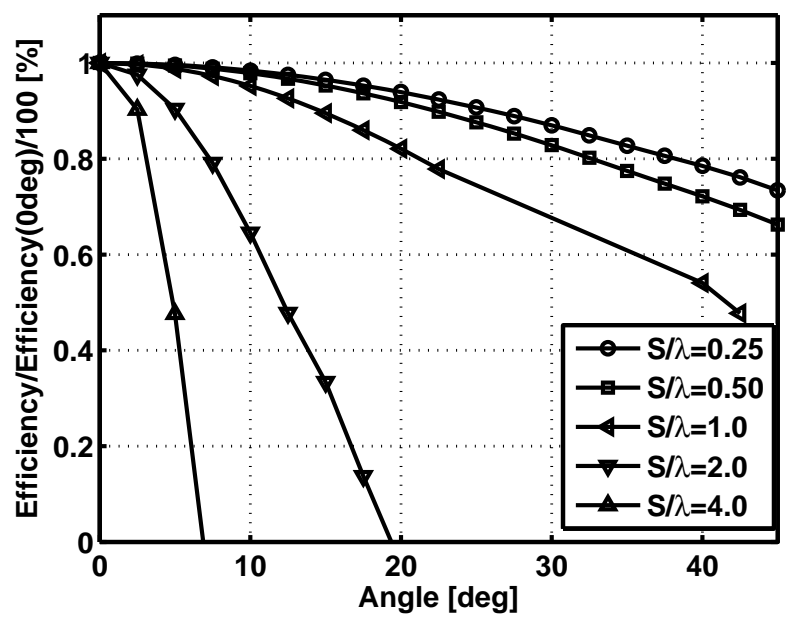

Figure 18: Wave Cancellation efficiency as a function of angle between WEC and Wave Crest for different WEC spans

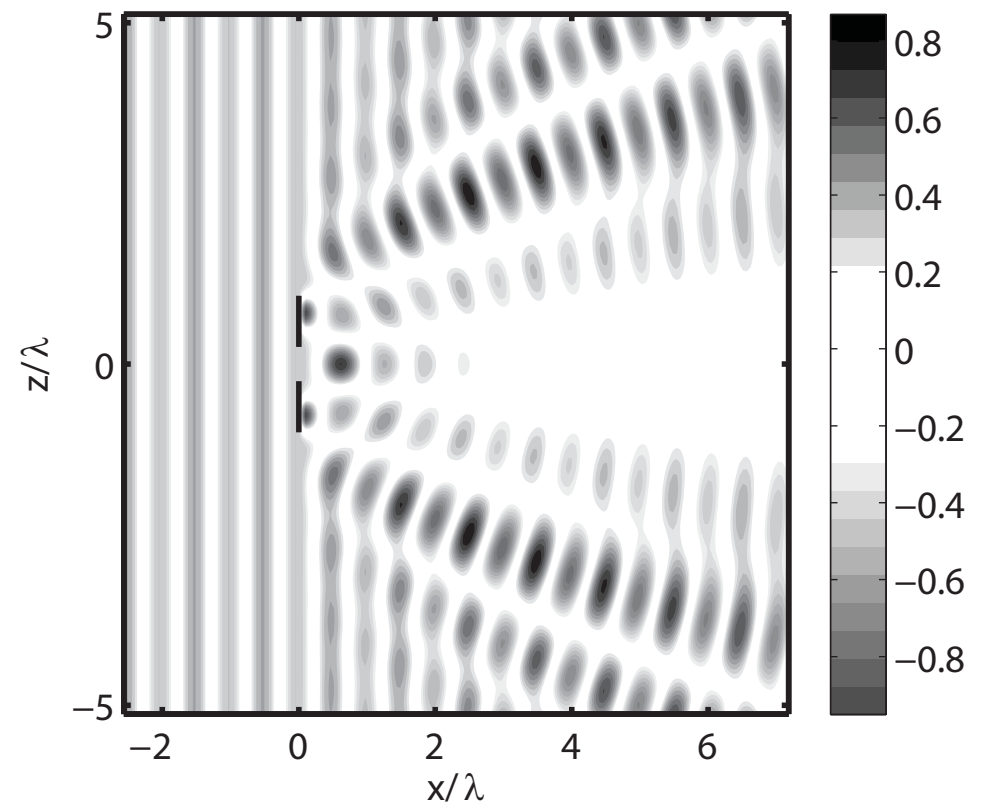

Figure 19: Surface Elevation during wave cancellation with a double WEC of size $S / \lambda=2$ and gap $G / S=0.5$ 


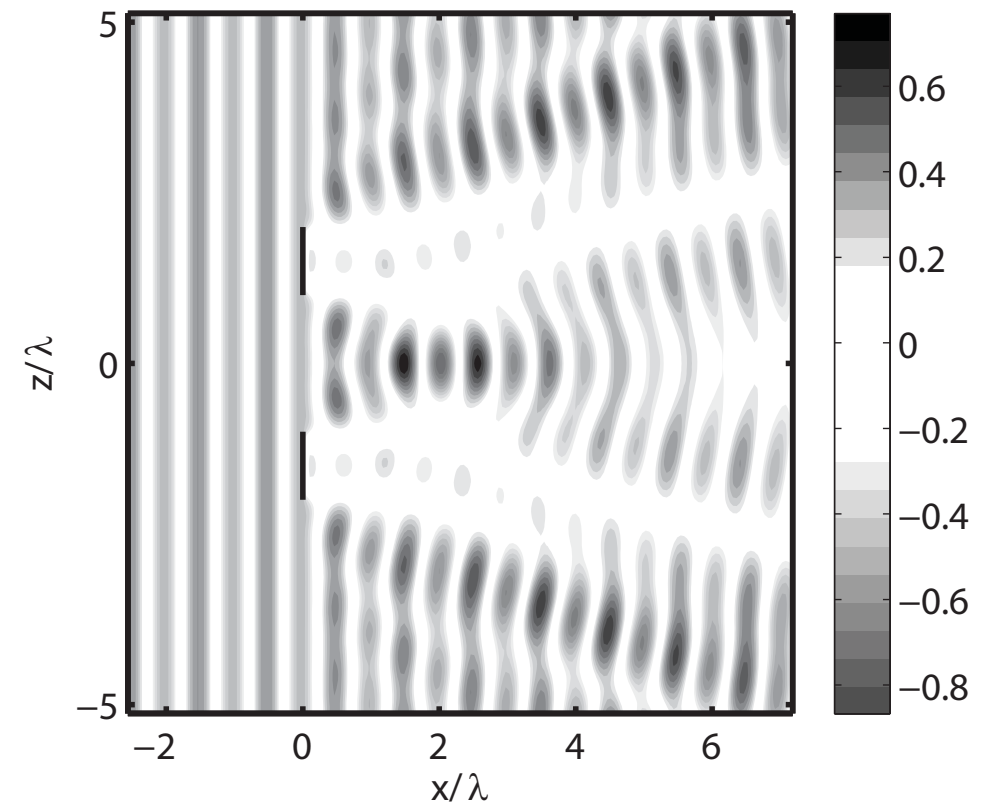

Figure 20: Surface Elevation during wave cancellation with a double WEC of size $S / \lambda=4$ and gap $G / S=0.5$

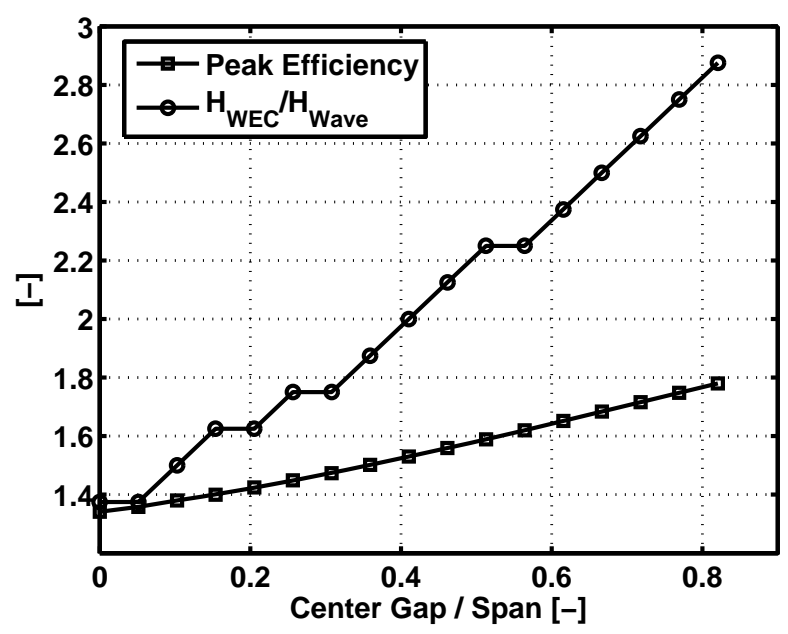

Figure 21: Maximum wave cancellation efficiency and height ratio as a function of non-dimensional gap size for a non-dimensional span of $s / \lambda=1$. 


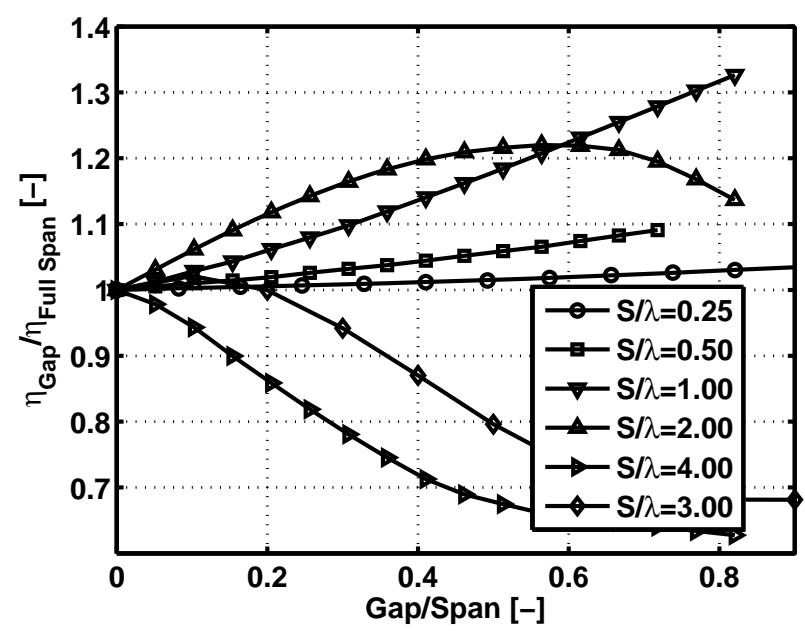

Figure 22: Wave Cancellation efficiency as a function of non-dimensional gap size for different WEC spans

of wave energy through the gap of a double WEC beyond a certain gap size as can be seen in the two surface elevation plots of Fig. 19 and Fig. 20.

The gap in a double cycloidal wave energy converter setup can either cause an increase in radiation efficiency, or a decrease. Sign and magnitude of this efficiency change depend in a nonlinear fashion on the non-dimensional parameters $S / \lambda$ and $G / S$. However, the magnitude of these efficiency changes was relatively limited when compared to the impact of the $S / \lambda$ ratio of a single WEC, where for small ratios of $S / \lambda$ an increase of more than $350 \%$ compared to the two dimensional results was found. In comparison, the effect of the center gap on efficiency as shown in Fig. 22 was limited to plus or minus $35 \%$ of the efficiency without any gap. Nonetheless the impact of gap size on WEC performance should be carefully considered in the design process to optimize the overall performance of the double cycloidal wave energy converter. More importantly, the required adjustment in WEC generated wave height needs to be part of the overall blade pitch control system to achieve optimal performance and avoid pitch control induced losses. These could be well beyond the efficiency changes shown here, which assumed optimal blade pitch control. The reason for the required adjustment in WEC generated wave height is the same as for the adjustment that had been made to the WEC wave height in considering a non-uniform spanwise lift distribution as reported in Fagley et al. [18]. There it was found that in order to compensate for smaller wave generation towards the tip of the hydrofoil the center wave height had to be increased in proportion to achieve optimal cancellation, so that the average wave height matched that of the incoming wave. Similarly, in the present investigation the existence of a gap in wave generation has to be compensated by larger wave height generation by the remaining hydrofoil spans. This is evident in Fig. 21 where the required height ratio approaches the value of $4 / \pi$ for small gaps, and increases with increasing gap size.

\section{Conclusions}

In order to understand and isolate the impact of radiation on the WEC performance, a simple three-dimensional radiation model was developed based on linear superposition of circular point wave sources with a azimuthal cosine modulation of the wave height. The model results agreed well with measured wave heights from a 1:10 scale experiment, and showed that wave radiation induced wave focusing increased the wave power that could be extracted beyond the two-dimensional limit. This indicated that while the WEC avoided the losses due to up-wave radiated waves suffered by typical symmetric point absorbers, it could nonetheless leverage the benefits of radiation induced wave focusing at small span to wave length ratios.

The numerical model was subsequently used to investigate the sensitivity of the WEC to angular misalignment between WEC shaft and incoming wave crests. The impact of misalignment on efficiency was found to be strongly dependent on the ratio between span of the WEC and incoming wavelength. For short spans, the reduction in power 
extraction efficiency was minor, while very large spans showed significant reductions in efficiency. This loss in efficiency could be attributed to a mismatch in phase between the incoming wave and the WEC, which increased with increasing span due to the larger displacement of the WEC blade tips relative to the incoming wave front.

A gap between the blades of a double WEC had a relatively minor effect on efficiency which depended on the gap size as well as the overall span to wavelength ratio. For small span to wavelength ratios up to $s / \lambda=2$ an increase in efficiency of up to $30 \%$ was found, while for larger spans the gap resulted in a decrease in efficiency.

\section{Acknowledgments}

The author would like to acknowledge the fruitful discussions with his colleagues at Atargis Energy, most importantly Dr. Seidel and Dr. Fagley. This material is partially based upon work supported by the Department of Energy under Award Number DE-EE0003635.

\section{Disclaimer}

This report was in part prepared as an account of work sponsored by an agency of the United States Government. Neither the United States Government nor any agency thereof, nor any of their employees, makes any warranty, express or implied, or assumes any legal liability or responsibility for the accuracy, completeness, or usefulness of any information, apparatus, product, or process disclosed, or represents that its use would not infringe privately owned rights. Reference herein to any specific commercial product, process, or service by trade name, trademark, manufacturer, or otherwise does not necessarily constitute or imply its endorsement, recommendation, or favoring by the United States Government or any agency thereof. The views and opinions of the author expressed herein do not necessarily state or reflect those of the United States Government or any agency thereof.

\section{References}

[1] Boyle G. Renewable Energy - Power for a sustainable future. Oxford University Press; 2004.

[2] Bedart R. Final summary report - offshore wave power feasibility demonstration project. Tech. Rep.; E2I EPRI Global, WP 009 - US Rev 1; 2005.

[3] Siegel SG, Fagley C, Nowlin S. Experimental wave termination in a 2d wave tunnel using a cycloidal wave energy converter. Applied Ocean Research 2012;38:92-99. doi:http://dx.doi.org/10.1016/j.apor.2012.07.003.

[4] Evans DV. A theory for wave-power absorption by oscillating bodies 1976;77(1):1-25.

[5] Betz A. Das maximum der theoretisch möglichen ausnützung des windes durch windmotoren. Zeitschrift für das gesamte Turbinenwesen 1920;26:307309.

[6] McCormick M. Ocean Wave Energy Conversion. John Wiley \& Sons; 1981.

[7] Mei CC. The Applied Dynamics of Ocean Surface Waves. John Wiley \& Sons, New York; 1983.

[8] Cruz J. Ocean wave energy: current status and future prepectives. Springer-Verlag; 2008.

[9] Salter SH. World progress in wave energy-1988. International journal of ambient energy 1989;10(1):3-24.

[10] Evans DV, Jeffrey DC, Salter SH, Taylor JRM. Submerged cylinder wave energy device: theory and experiment. Applied Ocean Research 1979;1(1):3-12.

[11] Hermans AJ, van Sabben E, Pinkster J. A device to extract energy from water waves. Applied Ocean Research Computational Mechanics Publications 1990; Vol. 12, No. 4:5.

[12] Marburg C. Investigation on a Rotating Foil for Wave Energy Conversion. Master's thesis; TU Delft; 1994.

[13] Siegel SG, Jeans T, McLaughlin T. Deep ocean wave energy conversion using a cycloidal turbine. Applied Ocean Research April 2011; Volume 33 Issue 2:110-119. doi:http://dx.doi.org/10.1016/j.apor.2011.01.004.

[14] Siegel S, Jeans T, McLaughlin T. Deep ocean wave cancellation using a cycloidal turbine. In: 62nd Annual Meeting of the American Physical Society, Division of Fluid Dynamics, Minneapolis, MN. 2009,.

[15] Jeans T, Siegel SG, Fagley C, Seidel J. Irregular deep ocean wave energy conversion using a cycloidal wave energy converter. In: 9th European Wave and Tidal Energy Conference (EWTEC),Southampton, UK, September 5th 9th. 2011,.

[16] Siegel SG, Fagley C, Roemer M, McLaughlin T. Experimental wave cancellation using a cycloidal wave energy converter. In: 9th European Wave and Tidal Energy Conference (EWTEC),Southampton, UK, September 5th 9th. 2011,.

[17] Siegel S, Fagley C, Roemer M, Mclaughlin T. Experimental investigation of irregular wave cancellation using a cycloidal wave energy converter. In: 31st International Conference on Ocean, Offshore and Arctic Engineering (OMAE). OMAE2012-83388; 2012,

[18] Fagley C, Siegel S, Seidel J. Wave cancellation experiments using a 1:10 scale cycloidal wave energy converter. In: 1st Asian Wave and Tidal Energy Conference (AWTEC) Jeju Island, Korea, November 27-30. 2012,

[19] Siegel SG, Fagley C, Seidel J. 3d wave diffraction efficiency of a double cycloidal wave energy converter. In: 10th European Wave and Tidal Energy Conference (EWTEC),Aalborg, DK, September 5th 9th. 2013,.

[20] Siegel SG. Wave climate scatter performance of a cycloidal wave energy converter. Applied Ocean Research 2014;submitted April:under review. 